\title{
Erratum
}

\section{Relation between the spin Hall conductivity and the spin Chern number for Dirac-like systems}

\author{
[Int. J. Geom. Methods Mod. Phys., Vol. 13, No. 1 (2016) \\ Article ID: 1550136 (17 pages), DOI: 10.1142/S0219887815501364] \\ Ömer F. Dayi* and Elif Yunt ${ }^{\dagger}$ \\ Physics Engineering Department, Faculty of Science and Letters \\ Istanbul Technical University, TR-34469 \\ Maslak-Istanbul, Turkey \\ *dayi@itu.edu.tr \\ †yunt@itu.edu.tr
}

Published 21 June 2016

Distribution functions were defined for negative energies instead of positive energies. However, this does not alter the results obtained in the article. Correct definitions are as follows.

- Beginning of Page 6: the theta-function at the Fermi energy $E_{F}: f=\theta\left(E_{F}-E\right)$. Thus, the anamolous Hall conductivity reads

$$
\sigma_{\mathrm{AH}}=e^{2} \int_{E<E_{F}} \frac{d^{2} p}{(2 \pi \hbar)^{2}} G_{x y} .
$$

- End of Page 6: Once this is done we can set the ground state distribution functions to unity by restricting our integrals to energies less than the Fermi energy.

- Beginning of Page 12: Therefore, $f$ restricts the integral to energies less than the Fermi energy and the spin Hall conductivity becomes... 